\title{
Cyanoacrylate injection versus band ligation for bleeding from cardiac varices along the lesser curvature of the stomach
}

\author{
Sang Jung Park ${ }^{1 *}$, Yong Kwon Kim², Yeon Seok Seo', Seung Woon Park', Han Ah Lee', Tae Hyung Kim', Sang Jun Suh', \\ Young Kul Jung', Ji Hoon Kim', Hyunggin An ${ }^{3}$, Hyung Joon Yim', Jae Young Jang², Jong Eun Yeon', Kwan Soo Byun', \\ and Soon $\mathrm{Ho} \mathrm{Um}^{1}$
}

'Department of Internal Medicine, Korea University College of Medicine; ${ }^{2}$ Department of Internal Medicine, Soonchunhyang University; ${ }^{3}$ Department of Biostatistics, Korea University College of Medicine, Seoul, Korea

Background/Aims: Practice guidelines recommend endoscopic band ligation (EBL) and endoscopic variceal obturation (EVO) for bleeding from esophageal varices and fundal varices, respectively. However, the optimal treatment for bleeding from cardiac varices along the lesser curvature of the stomach (GOV1) remains undefined. This retrospective study compared the efficacy between EBL and EVO for bleeding from GOV1.

Methods: Patients treated by EBL or EVO via cyanoacrylate injection for bleeding from GOV1 were enrolled. Patients diagnosed with hepatocellular carcinoma or treated with endoscopic injection sclerotherapy were excluded.

Results: The study included 91 patients treated for bleeding from GOV1. The mean age was $56.3 \pm 10.9$ years (mean \pm SD), and 78 of them (85.7\%) were men. Overall, 51 and 40 patients were treated with EBL and EVO, respectively. A trend for a higher hemostasis rate was noted in the EVO group (100\%) than in the EBL group (82.6\%, $P=0.078)$. Varices rebled in 15 patients during follow-up. The rebleeding rate was significantly higher in the EBL group than in the EVO group $(P=0.004)$. During follow-up, 13 patients died (11 in the EBL group and 2 in the EVO group); the survival rate was marginally significant between two groups $(P=0.050)$. The rebleeding-free survival rate was significantly higher in the EVO group than in the EBL group $(P=0.001)$.

Conclusions: Compared to EBL, EVO offered significantly lower rebleeding rates, significantly higher rebleeding-free survival rates, and a trend for higher hemostasis and survival rates. EVO appears to be the better therapeutic option for bleeding from GOV1. (Clin Mol Hepatol 2016;22:487-494)

Keywords: Varices; Liver cirrhosis; Endoscopy; Cyanoacrylate; Band ligation

\section{INTRODUCTION}

Bleeding from gastroesophageal varices (GOVs) is a common and severe complication of liver cirrhosis. It presents in approxi- mately $50 \%$ of patients with cirrhosis' and once it develops, it frequently bleeds in $10-15 \%$ patients per year. ${ }^{2}$ Although prognoses of patients with variceal bleeding have improved significantly over previous decades, mortality rates remain as high as $15-20 \% .^{3-5} \mathrm{In}$

\footnotetext{
Abbreviations:

$A L T$, alanine aminotransferase; $\mathrm{Cl}$, confidence interval; $\mathrm{EBL}$, endoscopic band ligation; EV, esophageal varices; EVO, endoscopic variceal obturation; GOV, gastrooesophageal varices; GV, gastric varices; HCC, hepatocellular carcinoma; HR, hazard ratio; INR, international normalized ratio; MELD, model for end-stage liver disease
}

\section{Corresponding author: Yeon Seok Seo}

Division of Gastroenterology and Hepatology, Department of Internal Medicine, Korea University College of Medicine, 73 Inchon-ro, Seongbukgu, Seoul 02841, Korea

Tel: +82-2-920-6608, Fax: +82-2-953-1943

E-mail: drseo@korea.ac.kr 
addition, rebleeding after hemostasis is very common if appropriate prophylactic treatment is not administered. ${ }^{3,6}$ Because of the poor prognosis of bleeding from GOVs, prompt and appropriate management of these patients is essential.

Gastric varices (GVs) occur in approximately $20 \%$ of patients with portal hypertension. ${ }^{7,8}$ Although bleeding from GVs occurs less frequently than from esophageal varices (EVs), it is usually more severe as compared to bleeding from EVs, with higher rebleeding and mortality rates. ${ }^{7,9,10}$ Because GVs are located deeper in the tissues, are usually larger, and are continuously exposed to gastric acid and pepsin as compared to EVs, treatment failure rates are higher in GV bleeding than in EV bleeding. ${ }^{11,12}$ Therefore, endoscopic band ligation (EBL), which is considered the treatment of choice for EV bleeding, is not recommended for GV bleeding. Instead, endoscopic injection of N-butyl-2-cyanoacrylate (endoscopic variceal obturation, EVO) is considered as the optimal endoscopic treatment for GV bleeding. ${ }^{13,14}$

GVs are usually classified as type 1 GOVs (GOV1), type 2 GOVs (GOV2), type 1 isolated GVs (IGV1), and type 2 IGVs (IGV2), according to the Sarin classification as follows. ${ }^{7}$ GOV1 appears as a continuation of EVs and extends for $2-5 \mathrm{~cm}$ below the gastroesophageal junction along the lesser curvature of the stomach, while GOV2 extend below the gastroesophageal junction into the fundus. ${ }^{7}$ Although GOV1 is the most common type of GVs (about $75 \%$ of $\mathrm{GVs})^{7}$ and a common site of GV bleeding ( $67 \%$ of all GV bleeding) ${ }_{1}^{13}$ there remain some controversies regarding the optimal treatment of bleeding from cardiac varices on the lesser curvature side of the stomach (GOV1). The Baveno $V$ consensus workshop recommends both EBL and EVO in bleeding from GOV1, ${ }^{15}$ while some experts suggest treatment in the same manner as when EVs are present. ${ }^{16-18}$ In the subgroup analysis of rebleeding rate from GOV1 in the randomized controlled trials for GV bleeding between EBL and EVO, 13,14 rebleeding from GOV1 did not differ significantly between EBL and EVO groups. ${ }^{5,14}$ However, in a recent meta-analysis, EVO was found to be superior to EBL for the prevention of rebleeding from GOV1. ${ }^{19} \mathrm{~A}$ recent randomized controlled trial showed similar efficacy between EBL and EVO for treating GOV1 bleeding, ${ }^{20}$ while a retrospective study showed a higher incidence of post-treatment ulcer bleeding and higher mortality rates in the EBL group as compared to those in the EVO group. ${ }^{21}$ This retrospective study aimed to evaluate EBL and EVO in order to identify the optimal endoscopic treatment for bleeding from GOV1 in patients with liver cirrhosis.

\section{MATERIAL AND METHODS}

\section{Patients}

Consecutive patients with bleeding from GOV1 who were treated with EBL or EVO between January 2006 and December 2015 were included in this study. Patients with coexistent hepatocellular carcinoma (HCC) or patients with bleeding from EVs, GOV2, IGV1, or IGV2 were excluded. Patients with GOV1 bleeding who were treated with endoscopic injection sclerotherapy were also excluded. Liver cirrhosis was diagnosed based on histology and/or imaging studies. The study protocol conformed to the ethical guidelines of the 1975 Helsinki Declaration and was approved by the Institutional Review Board. A waiver of consent was obtained and the patient records were anonymized and de-identified prior to analysis.

\section{Data collection}

Patient data for age, sex, and underlying liver disease (chronic hepatitis $B$, chronic hepatitis $C$, alcoholic liver disease, and others) were collected during hospitalization. Results of laboratory tests [platelet count, international normalized ratio (INR), serum alanine aminotransferase (ALT), bilirubin, albumin, and creatinine $(\mathrm{Cr})$ levels] were collected at enrollment. The Child-Pugh score was determined by applying Pugh's commonly used modification, which is based on the presence and severity of ascites and hepatic encephalopathy, prothrombin time prolongation, and serum bilirubin and albumin levels. ${ }^{22}$ The model for end-stage liver disease (MELD) score was calculated using the following equation: $9.57 \times \log _{\mathrm{e}}(\mathrm{Cr}, \mathrm{mg} / \mathrm{dL})+3.78 \times \log _{\mathrm{e}}$ (bilirubin, $\mathrm{mg} / \mathrm{dL}$ ) $+11.2 \times \log _{\mathrm{e}}$ $($ INR $)+6.43 .^{23}$ The minimum value was set at 1.0 for calculation purposes. The maximum serum $\mathrm{Cr}$ level considered in the above equation was $4.0 \mathrm{mg} / \mathrm{dL}$.

The size of the EVs and cardiac varices was measured at the initial endoscopy-before treatment with EBL; the size of the EVs was measured according to Beppu's classification. ${ }^{24}$ To measure the size of the cardiac varices, the diameter and scope were measured in the endoscopic pictures that had been taken before endoscopic treatment. The real diameter of the cardiac varices was calculated using the ratio of the measured diameter of the cardiac varices to the measured diameter of the scope, as well as the real diameter of the scope $(9.8$ mm; GIF-H260; Olympus Optical Co., Ltd, Tokyo, Japan).

The type of bleeding was classified into active bleeding, stig- 
mata, and nonactive bleeding as follows ${ }^{4}$ : active bleeding, a spurting or oozing from varices; stigmata, the presence of a white nipple, erosive spots, or adherent clots on varices; and nonactive bleeding, neither active bleeding nor stigmata. Control of bleeding was defined when there was no hematemesis, the hemoglobin level was stable without requiring blood transfusions, and the vital signs were stable (systolic blood pressure $>100 \mathrm{mmHg}$ and pulse rate $<100$ beats $/ \mathrm{min}$ ) for $24 \mathrm{~h}{ }^{4}{ }^{4}$ Follow-up data for rebleeding and mortality were collected until 2 year after enrollment. Rebleeding was defined as any occurrence of hematemesis or a decrease in the hemoglobin level, with fresh melena after the successful control of the initial bleeding. ${ }^{4}$

\section{Statistics}

Statistical analyses were performed using SPSS software version 20.0 (SPSS Inc., Chicago, IL, USA). Data were expressed as mean \pm standard deviation or number (\%). The Mann-Whitney U test and chi-square test were used to compare continuous and categorical variables, respectively. The cumulative rebleeding and mortality rates were determined by the Kaplan-Meier method, and the difference between groups was determined by the loglank test. The Cox proportion hazard model was utilized to analyze factors associated with rebleeding, mortality, and rebleedingfree mortality; significant factors $(P<1.0)$ in the univariate analysis were subjected to multivariate analysis to determine independent

Table 1. Baseline characteristics of patients with bleeding from GOV1 according to the type of endoscopic treatment

\begin{tabular}{|c|c|c|c|c|}
\hline & All patients $(n=91)$ & EBL group $(n=51)$ & EVO group $(n=40)$ & $P$-value \\
\hline Age, years & $56.3 \pm 10.9$ & $56.9 \pm 10.2$ & $55.5 \pm 11.8$ & 0.531 \\
\hline Male, n (\%) & 78 (85.7) & $44(86.3)$ & $34(85.0)$ & 0.863 \\
\hline Etiology, n (\%) & & & & 0.234 \\
\hline Alcoholic liver disease & $55(60.4)$ & $33(64.7)$ & $22(55.0)$ & \\
\hline Chronic hepatitis B & 27 (29.7) & $15(29.4)$ & $12(30.0)$ & \\
\hline Chronic hepatitis C & $3(3.3)$ & $2(3.9)$ & $1(2.5)$ & \\
\hline Others & $6(6.6)$ & $1(2.0)$ & $5(12.5)$ & \\
\hline Size of $E V, n(\%)$ & & & & 0.352 \\
\hline $\mathrm{F} 1$ & $14(15.4)$ & $9(17.6)$ & $5(12.5)$ & \\
\hline F2 & $54(59.3)$ & $32(62.7)$ & $22(55.0)$ & \\
\hline F3 & $23(25.3)$ & $10(19.6)$ & $13(32.5)$ & \\
\hline Size of GOV1, cm & $1.2 \pm 0.4$ & $1.2 \pm 0.4$ & $1.3 \pm 0.4$ & 0.100 \\
\hline Type of bleeding, n (\%) & & & & 0.213 \\
\hline Active bleeding & $39(42.9)$ & $23(45.1)$ & $16(40.0)$ & \\
\hline Stigmata & $42(46.2)$ & $25(49.0)$ & $17(42.5)$ & \\
\hline Nonactive bleeding & $10(11.0)$ & $3(5.9)$ & $7(17.5)$ & \\
\hline Platelet count, $\times 10^{9} / \mathrm{L}$ & $106.6 \pm 70.9$ & $118.8 \pm 83.4$ & $91.1 \pm 47.3$ & 0.065 \\
\hline INR & $1.7 \pm 0.7$ & $1.7 \pm 0.7$ & $1.6 \pm 0.5$ & 0.198 \\
\hline $\mathrm{ALT}, \mathrm{IU} / \mathrm{L}$ & $44.9 \pm 58.9$ & $51.7 \pm 74.6$ & $36.3 \pm 26.9$ & 0.218 \\
\hline Bilirubin, mg/dL & $2.7 \pm 3.6$ & $2.8 \pm 3.5$ & $2.7 \pm 3.9$ & 0.918 \\
\hline Albumin, g/dL & $2.9 \pm 0.5$ & $2.8 \pm 0.5$ & $3.0 \pm 0.6$ & 0.114 \\
\hline Creatinine, mg/dL & $1.0 \pm 0.3$ & $1.0 \pm 0.4$ & $0.9 \pm 0.3$ & 0.414 \\
\hline Child-Pugh score & $7.6 \pm 1.8$ & $7.3 \pm 1.7$ & $7.8 \pm 1.9$ & 0.195 \\
\hline MELD score & $15.2 \pm 5.9$ & $15.6 \pm 6.4$ & $14.6 \pm 5.3$ & 0.432 \\
\hline
\end{tabular}

ALT, alanine aminotransferase; EBL, endoscopic band ligation; EV, esophageal varices; EVO, endoscopic variceal obturation; GOV1, gastroesophageal varices type 1; INR, international normalized ratio; MELD, model for end-stage liver disease. 
predictive factors. All tests were two-tailed, and $P$ values $<0.05$ were considered statistically significant.

\section{RESULTS}

\section{Baseline characteristics}

Overall, 91 patients with bleeding from GOV1 were enrolled in this study. Table 1 shows the baseline characteristics of the enrolled patients. The mean age was $56.3 \pm 10.9$ years and 78 patients (85.7\%) were men. Alcoholic liver disease was the most common underlying liver disease (55 patients, $60.4 \%$ ), followed by chronic hepatitis B virus infection (27 patients, 29.7\%). ChildPugh and MELD scores were $7.6 \pm 1.8$ and $15.2 \pm 5.9$, respectively. Active bleeding was noted in the first endoscopy in 39 patients (42.9\%). Patients with active bleeding was comparable between EBL and EVO groups ( $45.1 \%$ vs. $40.0 \%, P=0.626$ ), however there was a trend for higher proportion of patients with active bleeding or stigmata in the EBL group ( $94.1 \%$ vs. $82.5 \%, P=0.079)$.

\section{Control of bleeding}

Among 91 patients, 51 (56.0\%) and 40 (44.0\%) patients were treated with EBL and EVO, respectively. Baseline characteristics were comparable between the two groups (Table 1). Among 39 patients with active bleeding at the initial endoscopy, 23 (59.0\%) and $16(41.0 \%)$ patients were treated with EBL and EVO, respectively. Bleeding was successfully controlled in 35 patients (89.7\%). A trend for higher hemostasis rates was noted in the EVO group (16 patients, 100\%) as compared to the EBL group (19 patients, $82.6 \% ; P=0.078$ ).

\section{Rebleeding}

After successful hemostasis, varices rebled in 15 patients during follow-up (14 patients in the EBL group and 1 patient in the EVO group) and the cumulative rebleeding rates at $6,12,18$, and 24 months were $10.3 \%, 19.9 \%, 24.2 \%$, and $26.6 \%$, respectively. The rebleeding rate was significantly higher in the EBL group as compared to those in the EVO group ( $P=0.004$ ) (Fig. 1). Rebleeding rates at $6,12,18$, and 24 months were $15.1 \%, 30.8 \%, 37.5 \%$, and $41.4 \%$, respectively, in the EBL group, and $3.6 \%, 3.6 \%$, $3.6 \%$, and $3.6 \%$, respectively, in the EVO group.

On univariate analysis, the type of treatment, INR, serum bilirubin

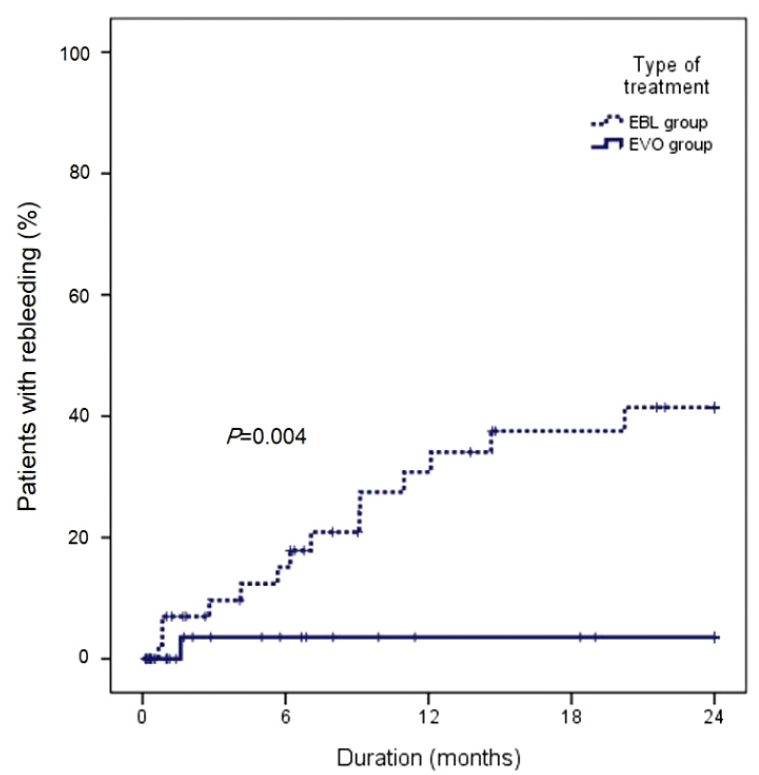

Figure 1. Cumulative rebleeding rates in patients with GOV1 bleeding. EBL, endoscopic band ligation; EVO, endoscopic variceal obturation.

level, and MELD score were associated with rebleeding (Table 2). Because INR and bilirubin level were included in the MELD score, these variables were not used in multivariate analysis. Treatment with $E B L(\beta, 2.170$; hazard ratio [HR], 8.758; 95\% confidence interval $[\mathrm{Cl}], 1.136-67.501 ; P=0.037)$ and high MELD score $(\beta$, $0.171 ; \mathrm{HR}, 1.186 ; 95 \% \mathrm{Cl}, 1.086-1.296 ; P<0.001)$, were independent predictors for rebleeding in multivariate analyses (Table 3).

\section{Mortality}

During follow-up, 13 patients died (11 and 2 patients in the EBL and EVO groups, respectively). The cause of death was variceal bleeding in 9 patients, liver failure in 3 patients, and sepsis in 1 patient. Cumulative survival rates at $6,12,18$, and 24 months were $88.9 \%, 83.6 \%, 81.7 \%$, and $81.7 \%$, respectively. As compared to the EBL group, higher survival rate was noted in the EVO group with statistically marginal significance $(85.5 \%$ vs. $93.1 \%$, $77.2 \%$ vs. $93.1 \%, 74.2 \%$ vs. $93.1 \%$, and $74.2 \%$ vs. $93.1 \%$ at 6 , 12,18 , and 24 months, respectively; $P=0.050$ ) (Fig. 2).

The type of bleeding, INR, serum bilirubin, albumin, and creatinine levels, and Child-Pugh and MELD scores were associated with mortality and there was a trend for higher mortality rate in the EBL group on univariate analysis (Table 2). Because INR and serum bilirubin, albumin, and creatinine levels were included in the Child-Pugh and/or MELD scores, these variables were not used in multivariate analysis. The MELD score $(\beta, 0.203 ; \mathrm{HR}$, 
Sang Jung Park, et al.

Optimal therapy for cardiac variceal bleeding

Table 2. Results of univariate Cox regression analyses of rebleeding, mortality, rebleeding, and mortality rates in patients with GOV1 bleeding

\begin{tabular}{|c|c|c|c|c|}
\hline & Datingorunit & & HR (95\% Cl); $P$-value & \\
\hline & Katıng or unit & Rebleeding & Mortality & Rebleeding or mortality \\
\hline Age & years & 0.968 (0.924-1.013); 0.161 & $0.985(0.937-1.036) ; 0.565$ & $0.983(0.948-1.020) ; 0.369$ \\
\hline Sex & 1=female; $2=$ male & $0.803(0.226-2.848) ; 0.734$ & $26.239(0.041-16951.363) ; 0.322$ & $1.307(0.388-4.402) ; 0.660$ \\
\hline Etiology & 1=nonalcoholic; $2=$ alcoholic & $2.491(0.702-8.843) ; 0.158$ & $1.011(0.330-3.097) ; 0.985$ & $1.777(0.700-4.514) ; 0.226$ \\
\hline Size of EV & $1=F 1 ; 2=F 2 ; 3=F 3$ & $0.627(0.301-1.307) ; 0.213$ & $0.986(0.436-2.227) ; 0.972$ & $0.682(0.375-1.240) ; 0.356$ \\
\hline Size of CV & $\mathrm{cm}$ & $0.598(0.151-2.358) ; 0.462$ & $1.052(0.259-4.283) ; 0.943$ & $0.819(0.281-2.385) ; 0.714$ \\
\hline Type of bleeding & $\begin{array}{l}1=\text { not active; } \\
2=\text { active bleeding }\end{array}$ & $2.325(0.826-6.540) ; 0.110$ & 4.807 (1.322-17.480); 0.017 & 2.280 (1.194-6.659); 0.018 \\
\hline Type of treatment & $1=E V O ; 2=E B L$ & $10.975(1.443-83.495) ; 0.021$ & 4.008 (0.888-18.093); 0.071 & $8.203(1.923-35.001) ; 0.004$ \\
\hline Platelet count & $\times 10^{9} / \mathrm{L}$ & $1.001(0.993-1.010) ; 0.819$ & $0.989(0.976-1.004) ; 0.140$ & $0.996(0.987-1.004) ; 0.322$ \\
\hline INR & ratio & $2.453(1.198-5.021) ; 0.014$ & $2.734(1.662-4.496) ;<0.001$ & $2.729(1.758-4.237) ;<0.001$ \\
\hline ALT & $I U / L$ & $1.003(0.997-1.009) ; 0.386$ & $0.990(0.964-1.016) ; 0.459$ & $1.001(0.995-1.007) ; 0.672$ \\
\hline Bilirubin & $\mathrm{mg} / \mathrm{dL}$ & $1.347(1.165-1.557) ;<0.001$ & $1.215(1.114-1.326) ;<0.001$ & $1.219(1.123-1.325)_{i}<0.001$ \\
\hline Albumin & $\mathrm{g} / \mathrm{dL}$ & $0.874(0.306-2.493) ; 0.801$ & $0.266(0.106-0.665) ; 0.005$ & $0.450(0.210-0.965) ; 0.040$ \\
\hline Creatinine & $\mathrm{mg} / \mathrm{dL}$ & 3.118 (0.600-16.201); 0.176 & 7.529 (2.003-28.300); 0.003 & 4.201 (1.307-13.496); 0.016 \\
\hline Child-Pugh score & score & $1.116(0.834-1.492) ; 0.461$ & $1.570(1.190-2.070) ; 0.001$ & $1.321(1.066-1.636) ; 0.011$ \\
\hline MELD score & score & $1.214(1.111-1.328) ;<0.001$ & $1.262(1.159-1.374) ;<0.001$ & $1.228(1.148-1.314) ;<0.001$ \\
\hline
\end{tabular}

ALT, alanine aminotransferase; $\mathrm{Cl}$, confidence interval; EBL, endoscopic band ligation; EV, esophageal varices; EVO, endoscopic variceal obturation; GOV1, gastroesophageal varices type 1; HR, hazard ratio; INR, international normalized ratio; MELD, model for end-stage liver disease.

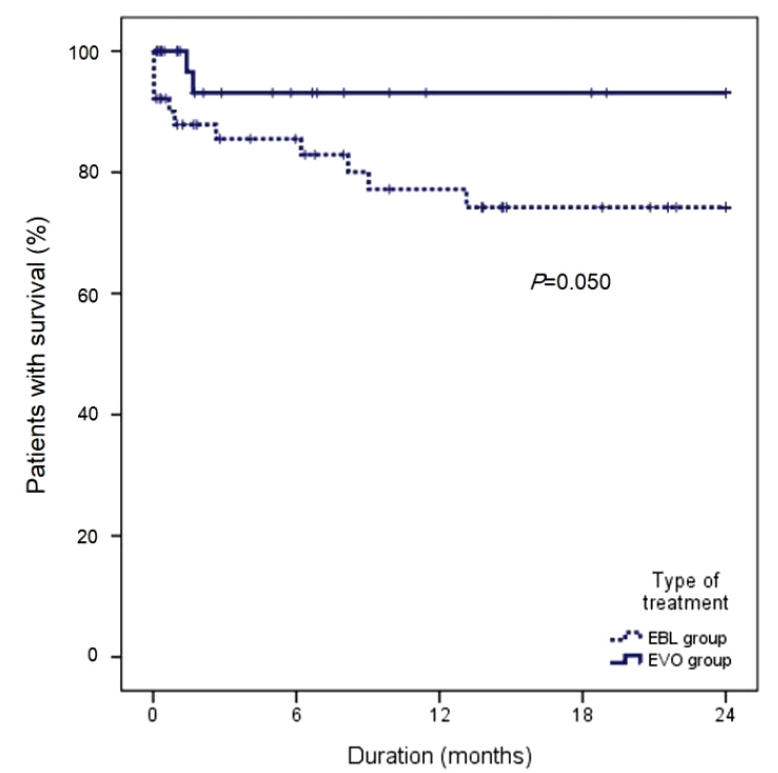

Figure 2. Cumulative survival rates in patients with GOV1 bleeding. EBL, endoscopic band ligation; EVO, endoscopic variceal obturation.

$1.225 ; 95 \% \mathrm{Cl}, 1.075-1.397 ; P=0.002)$ was the only independent predictor for mortality in patients with GOV1 bleeding. The type of bleeding ( $\beta, 0.501 ; \mathrm{HR}, 1.666 ; 95 \% \mathrm{Cl}, 0.415-6.688 ; P=0.472)$, type of treatment $(\beta, 0.866 ; \mathrm{HR}, 2.376 ; 95 \% \mathrm{Cl}, 0.457-12.361$;
$P=0.304)$, and Child-Pugh score $(\beta, 0.031 ; \mathrm{HR}, 1.031 ; 95 \% \mathrm{Cl}$, 0.595-1.786; $P=0.913$ ) were not associated with mortality in multivariate analysis (Table 3 ).

\section{Rebleeding-free survival}

During follow-up, 23 patients died or rebled (21 and 2 patients in the EBL and EVO groups, respectively). Cumulative rebleedingfree survival rates at $6,12,18$, and 24 months were $83.1 \%$, $71.1 \%, 67.2 \%$, and $65.0 \%$, respectively. The rebleeding-free survival rates were significantly higher in the EVO group $193.1 \%$, $93.1 \%, 93.1 \%$, and $93.1 \%$ at $6,12,18$, and 24 months, respectively) as compared to those in the EBL group $(76.1 \%, 57.6 \%$, $51.6 \%$, and $48.2 \%$ at $6,12,18$, and 24 months, respectively; $P=0.001$ ) (Fig. 3).

The type of bleeding, type of treatment, INR, serum bilirubin, albumin, and creatinine levels, and Child-Pugh and MELD scores were associated with rebleeding-free survival (Table 2). Because INR and serum bilirubin, albumin, and creatinine levels were included in the Child-Pugh and/or MELD scores, these variables were not included in multivariate analysis. Treatment with EBL $(\beta, 1.637 ; \mathrm{HR}$, 5.139; 95\% Cl, 1.104-23.095; $P=0.033)$ and high MELD score $(\beta$, 0.210; HR, 1.233; $95 \% \mathrm{Cl}, 1.104-1.377 ; P<0.001)$ were independent 
Table 3. Results of multivariate Cox regression analyses of rebleeding, mortality, rebleeding, and mortality rates in patients with GOV1 bleeding

\begin{tabular}{|c|c|c|c|c|}
\hline & \multirow{2}{*}{ Rating or unit } & \multicolumn{3}{|c|}{ HR (95\% Cl); P-value } \\
\hline & & Rebleeding & Mortality & Rebleeding or mortality \\
\hline Type of bleeding & $\begin{array}{l}1=\text { not active; } \\
2=\text { active bleeding }\end{array}$ & N/A & $1.666(0.415-6.688) ; 0.472$ & $1.225(0.471-3.185) ; 0.678$ \\
\hline Type of treatment & $1=\mathrm{EVO} ; 2=\mathrm{EBL}$ & $8.758(1.136-67.501) ; 0.037$ & $2.376(0.457-12.361) ; 0.304$ & 5.139 (1.104-23.095); 0.033 \\
\hline Child-Pugh score & score & $\mathrm{N} / \mathrm{A}$ & $1.031(0.595-1.786) ; 0.913$ & $0.842(0.543-1.304) ; 0.440$ \\
\hline MELD score & score & $1.186(1.086-1.296) ;<0.001$ & 1.225 (1.075-1.397); 0.002 & $1.233(1.104-1.377) ;<0.001$ \\
\hline
\end{tabular}

$\mathrm{Cl}$, confidence interval; EBL, endoscopic band ligation; EVO, endoscopic variceal obturation; GOV1, gastroesophageal varices type 1; HR, hazard ratio; MELD, model for end-stage liver disease; N/A, not applicable.

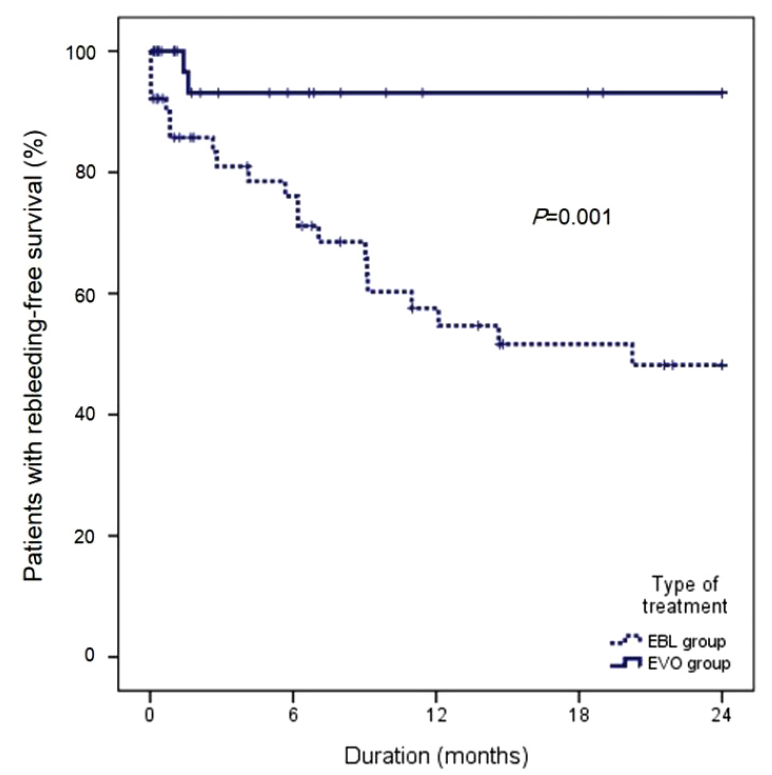

Figure 3. Cumulative rebleeding-free survival rates in patients with GOV1 bleeding. EBL, endoscopic band ligation; EVO, endoscopic variceal obturation.

predictors for rebleeding or mortality in patients with GOV1 bleeding, while type of bleeding $(\beta, 0.203 ; \mathrm{HR}, 1.225 ; 95 \% \mathrm{Cl}, 0.471$ 3.185; $P=0.678)$ and Child-Pugh score $(\beta,-0.173 ; H R, 0.842 ; 95 \%$ $\mathrm{Cl}, 0.543-1.304 ; P=0.440)$ were not associated with rebleedingfree survival in multivariate analysis (Table 3 ).

\section{DISCUSSION}

Recent practice guidelines recommend EBL for treating bleeding from EVs, while they recommend EVO for treating bleeding from GOV2 or IGV1. ${ }^{15}$ However, the optimal endoscopic treatment for GOV1 remains controversial. This controversy might be caused by the characteristics of GOV1. GOV1 shows characteristics of EVs as well as fundal varices. GOV1 is similar to EVs in terms of the size and the route of portal blood flow to the varices, while it is similar to fundal varices in terms of location. In patients with portal hypertension, the portal blood flow is reversed through the right and left gastric veins around the cardia in EVs and GOV1, while it is through short and distal gastric vein around the gastric fundus in fundal varices. ${ }^{18}$ In addition, the size of GOV1 is usually smaller than that of fundal varices. Therefore, some experts recommend EBL for bleeding from GOV1. ${ }^{16-18}$ However, GOV1 is located in the stomach, the same location as with GOV2, where the underlying submucosal layer is thicker than that in the esophagus, and is continuously exposed to gastric acid and pepsin. ${ }^{12}$ Therefore, the incidence of bleeding from EBL-induced ulcers may be more frequent in the stomach than in the esophagus. ${ }^{21}$ In addition, because of the thicker underlying submucosal layer over the varices in the stomach, the size of the varices may be underestimated on endoscopic observation. EBL is usually not recommended for large varices (diameter $>2 \mathrm{~cm}$ ) because of the limited diameter of the ligator. ${ }^{25} \mathrm{~A}$ thicker underlying submucosal layer could also interrupt complete suction of the variceal column into the device before ligation. These characteristics could increase the risk of treatment failure by EBL.

In our study, we noted a trend for higher hemostasis rate in 39 patients with active bleeding in the EVO group (100\% vs. $82.6 \%$ in the EBL group, $P=0.078$ ). This result is consistent with those of two previous studies, ${ }^{13,20}$ while the hemostasis rate did not differ between EBL and EVO in another study (Table 4). ${ }^{21}$ Since statistical non-significance could be due to relatively small number of patients with active bleeding in each study, the data of our study and those previous three studies ${ }^{13,20,21}$ were pooled together. Accordingly, 292 patients were treated, wherein EBL and EVO was used in 133 and 159 patients, respectively. In this pooled data, the hemostasis rate was significantly higher in the EVO group (144 of 159 patients, $90.6 \%$ ) than in the EBL group (107 of 133 pa- 
Sang Jung Park, et al.

Optimal therapy for cardiac variceal bleeding

Table 4. Summary of previous studies that have compared EBL and EVO for GOV1 bleeding

\begin{tabular}{|c|c|c|c|c|c|}
\hline $\begin{array}{l}\text { First author } \\
\text { (Year) }\end{array}$ & Study design & $\begin{array}{l}\text { Number of pts } \\
\text { (EBL vs. EVO) }\end{array}$ & $\begin{array}{l}\text { Control of active bleeding } \\
\text { (EBL vs. EVO) }\end{array}$ & $\begin{array}{l}\text { Rebleeding } \\
\text { (EBL vs. EVO) }\end{array}$ & $\begin{array}{c}\text { Mortality } \\
\text { (EBL vs. EVO) }\end{array}$ \\
\hline Lo et al. ${ }^{13}$ & Prospective RCT & 20 vs. 21 & $43 \%$ vs. $85 \%(P=0.08)$ & $55 \%$ vs. $26 \%(P=0.14)$ & N.A. \\
\hline Tan et al. ${ }^{14}$ & Prospective RCT & 26 vs. 27 & N.A. & $42.3 \%$ vs. $25.9 \%(P=N S)$ & N.A. \\
\hline El Amin et al. ${ }^{20}$ & Prospective RCT & 75 vs. 75 & $81 \%$ vs. $91 \%(P=0.07)$ & $16 \%$ vs. $6 \%(P=\mathrm{NS})$ & $1.3 \%$ vs. $6.6 \%(P=N S)$ \\
\hline Lo et al. ${ }^{21}$ & Retrospective study & 44 vs. 118 & $85 \%$ vs. $89 \%(P=0.70)$ & $6 \%$ vs. $6 \%(P=\mathrm{NS})$ & $23 \%$ vs. $14 \%(P<0.001)$ \\
\hline This study & Retrospective study & 51 vs. 40 & $82.6 \%$ vs. $100 \%(P=0.078)$ & $15.1 \%$ vs. $3.6 \%(P=0.004)$ & $14.5 \%$ vs. $6.9 \%(P=0.050)$ \\
\hline
\end{tabular}

EBL, endoscopic band ligation; EVO, endoscopic variceal obturation; N.A., not available; NS, not significant; RCT, randomized controlled trial.

tients, $80.5 \% ; P=0.013)$. Although this analysis is not sufficient to confirm that EVO is superior to EBL for hemostasis of active bleeding from GOV1, it suggests that the superior efficacy of EVO over EBL may be confirmed in a large-scale study.

Four previous studies ${ }^{13,14,20,21}$ compared rebleeding after hemostasis of bleeding from GOV1. Rebleeding rates did not differ between EBL and EVO in three studies, ${ }^{13,14,21}$ while one study showed a trend for higher rebleeding rates in the EBL group than in the EVO group $(16.0 \%$ vs. $6.7 \%, P=0.071) .{ }^{20}$ Although a previous study suggested that rebleeding rate did not differ between EBL and EVO, the incidence of ulcer bleeding from GOV1 and the number of patients who died due to bleeding from GOV1 was significantly higher in the EBL group. ${ }^{21}$ In the present study, the rebleeding rate was significantly higher in the EBL group than in the EVO group ( $P=0.004)$ and the type of treatment was one of the independent predictive factors for rebleeding in multivariate analysis. Our results suggest that EVO is superior to EBL for the prevention of rebleeding in patients with bleeding from GOV1.

This study showed a trend for higher survival rates in the EVO group as compared to those in the EBL group $(P=0.050)$, while the rebleeding-free survival rate was significantly higher in the EVO group ( $P=0.001)$. Furthermore, similar to the results of multivariate analysis for rebleeding, the type of treatment and the MELD score were independent predictors for rebleeding-free survival in patients with bleeding from GOV1. One previous study showed a trend for higher mortality rates in the EVO group than in the EBL group ( $6.7 \%$ vs. $1.3 \%, P=0.096){ }^{20}$ while the mortality rate within 42 days was significantly lower in the EVO group (14\% vs. $23 \%, P<0.001){ }^{21}$ This discrepancy in the treatment's effect on survival among studies could be due to different etiology and patient health status including remnant liver function of the patients included in each study. In addition, this discrepancy could be explained as follows: previous studies excluded patients with HCC only when it progressed to the advanced stage. Therefore, some number of patients with non-advanced HCC were included in their studies, and this may have affected survival rates because the prognosis of patients with HCC differs considerably from that of those without HCC. In contrast, in the present study, all patients with HCC were excluded.

In conclusion, as compared to EBL, EVO showed significantly lower rebleeding rates, significantly higher rebleeding-free survival rates, and a trend for higher hemostasis and survival rates. EVO is considered to be a better therapeutic option for bleeding from GOV1.

\section{Conflicts of Interest}

The authors have no conflicts to disclose.

\section{REFERENCES}

1. Garcia-Tsao G, Sanyal AJ, Grace ND, Carey W; Practice Guidelines Committee of the AASLD; Practice Parameters Committee of the ACG. Prevention and management of gastroesophageal varices and variceal hemorrhage in cirrhosis. Hepatology 2007;46:922-938.

2. North Italian Endoscopic Club for the Study and Treatment of Esophageal Varices. Prediction of the first variceal hemorrhage in patients with cirrhosis of the liver and esophageal varices. A prospective multicenter study. N Engl J Med 1988;319:983-989.

3. Graham DY, Smith JL. The course of patients after variceal hemorrhage. Gastroenterology 1981;80:800-809.

4. D'Amico G, De Franchis R; Cooperative Study Group. Upper digestive bleeding in cirrhosis. Post-therapeutic outcome and prognostic indicators. Hepatology 2003;38:599-612.

5. Stokkeland K, Brandt L, Ekbom A, Hultcrantz R. Improved prognosis for patients hospitalized with esophageal varices in Sweden 1969 2002. Hepatology 2006;43:500-505.

6. Baker LA, Smith C, Lieberman G. The natural history of esophageal varices; a study of 115 cirrhotic patients in whom varices were diagnosed prior to bleeding. Am J Med 1959;26:228-237.

7. Sarin SK, Lahoti D, Saxena SP, Murthy NS, Makwana UK. Prevalence, classification and natural history of gastric varices: a long-term 
follow-up study in 568 portal hypertension patients. Hepatology 1992;16:1343-1349.

8. Thakeb F, Salem SA, Abdallah M, el Batanouny M. Endoscopic diagnosis of gastric varices. Endoscopy 1994;26:287-291.

9. de Franchis $R$, Primignani M. Natural history of portal hypertension in patients with cirrhosis. Clin Liver Dis 2001;5:645-663.

10. Trudeau W, Prindiville T. Endoscopic injection sclerosis in bleeding gastric varices. Gastrointest Endosc 1986;32:264-268.

11. Kim MY, Um SH, Baik SK, Seo YS, Park SY, Lee Jl, et al. Clinical features and outcomes of gastric variceal bleeding: retrospective Korean multicenter data. Clin Mol Hepatol 2013;19:36-44.

12. Qureshi W, Adler DG, Davila R, Egan J, Hirota W, Leighton J, et al. ASGE Guideline: the role of endoscopy in the management of variceal hemorrhage, updated July 2005. Gastrointest Endosc 2005;62:651-655.

13. Lo GH, Lai KH, Cheng JS, Chen MH, Chiang HT. A prospective, randomized trial of butyl cyanoacrylate injection versus band ligation in the management of bleeding gastric varices. Hepatology 2001;33:1060-1064.

14. Tan PC, Hou MC, Lin HC, Liu TT, Lee FY, Chang FY, et al. A randomized trial of endoscopic treatment of acute gastric variceal hemorrhage: N-butyl-2-cyanoacrylate injection versus band ligation. Hepatology 2006;43:690-697.

15. de Franchis R; Baveno V Faculty. Revising consensus in portal hypertension: report of the Baveno $V$ consensus workshop on methodology of diagnosis and therapy in portal hypertension. J Hepatol 2010;53:762-768.

16. Sarin SK, Mishra SR. Endoscopic therapy for gastric varices. Clin Liver Dis 2010;14:263-279.
17. Garcia-Tsao G, Bosch J. Management of varices and variceal hemorrhage in cirrhosis. N Engl J Med 2010;362:823-832.

18. Ryan BM, Stockbrugger RW, Ryan JM. A pathophysiologic, gastroenterologic, and radiologic approach to the management of gastric varices. Gastroenterology 2004;126:1175-1189.

19. Qiao W, Ren Y, Bai Y, Liu S, Zhang Q, Zhi F. Cyanoacrylate Injection Versus Band Ligation in the Endoscopic Management of Acute Gastric Variceal Bleeding: Meta-Analysis of Randomized, Controlled Studies Based on the PRISMA Statement. Medicine (Baltimore) 2015;94:e1725.

20. El Amin H, Abdel Baky L, Sayed Z, Abdel Mohsen E, Eid K, Fouad $Y$, et al. A randomized trial of endoscopic variceal ligation versus cyanoacrylate injection for treatment of bleeding junctional varices. Trop Gastroenterol 2010;31:279-284.

21. Lo GH, Lin CW, Perng DS, Chang CY, Lee CT, Hsu CY, et al. A retrospective comparative study of histoacryl injection and banding ligation in the treatment of acute type 1 gastric variceal hemorrhage. Scand J Gastroenterol 2013;48:1198-1204.

22. Pugh RN, Murray-Lyon IM, Dawson JL, Pietroni MC, Williams R. Transection of the oesophagus for bleeding oesophageal varices. $\mathrm{Br}$ J Surg 1973;60:646-649.

23. Wiesner R, Edwards E, Freeman R, Harper A, Kim R, Kamath $P$, et al. Model for end-stage liver disease (MELD) and allocation of donor livers. Gastroenterology 2003;124:91-96.

24. Beppu K, Inokuchi K, Koyanagi N, Nakayama S, Sakata H, Kitano S, et al. Prediction of variceal hemorrhage by esophageal endoscopy. Gastrointest Endosc 1981;27:213-218.

25. Garcia-Pagán JC, Barrufet M, Cardenas A, Escorsell A. Management of gastric varices. Clin Gastroenterol Hepatol 2014;12:919-928. 